\title{
Hepatoprotective effect of various antioxidants in the pathogenesis of opisthorchiasis
}

\author{
O. Zaparina ${ }^{1 *}$, A. Kovner ${ }^{1}$, A. Rakhmetova ${ }^{2}$, M. Pakharukova ${ }^{1,2}$, V. Mordvinov ${ }^{1}$ \\ ${ }^{1}$ Institute of Cytology and Genetics SB RAS, Novosibirsk, Russia \\ ${ }^{2}$ Novosibirsk State University, Novosibirsk, Russia \\ *e-mail: zp.oksana.93@gmail.com
}

Key words: opisthorchiasis, inflammation, reactive oxygen species, antioxidants, liver flukes

Motivation and Aim: Opisthorchis felineus (liver fluke) is endemic to the territories of Europe and Asia the trematode of the family Opisthorchiidae, which infests the hepatobiliary system of fish-eating mammals including humans, causing opisthorchiasis. Pathogenesis of opisthorchiasis is accompanied by structural and functional disorders of the liver, including the formation of foci of chronic inflammation, cholestasis, cholecystitis and precancerous changes in the epithelium of the bile ducts. The mechanisms of development of this disease have not been studied; however, increased production of reactive oxygen species, which is accompanied by chronic inflammation, probably plays an important role in the pathogenesis of opisthorchiasis. The aim of the research was to study the effect of antioxidants of different mechanisms of action (natural antioxidant resveratrol and mitochondrial antioxidant SKQ1 (10- (6'-Plastoquinonyl) decyltriphenylphosphonium)) on the structural and functional state of the liver and to assess the possible role of oxidative stress in the pathogenesis of opisthorchiasis.

Methods and Algorithms: Experimental opisthorchiasis in vivo on golden hamsters M. auratus (1 and 3 months); semi-quantitative analysis of histological slides (with hematoxylin-eosin and Van-Gieson staining); Western blot analysis; blood biochemistry. Results: Pathological changes in the liver, including inflammation, dysplasia, metaplasia, proliferation of the bile duct epithelium and periductal fibrosis were quantified. Moreover, the content of markers of proliferation and dysplasia of cholangiocytes, as well as markers of inflammation were assessed. In addition, the activity of liver transaminases, cholesterol and bilirubin was evaluated. The hepatoprotective effect of antioxidants was demonstrated irrespective of the mechanism of their action. An improvement in the biochemical parameters of the liver, a decrease in dysplasia of the bile duct epithelium and a decrease in markers of inflammation on the background of experimental opisthorchiasis was demonstrated.

Conclusion: Oxidative stress plays an important role in the pathogenesis of opisthorchiasis. Antioxidants, regardless of the mechanism of their action, have a hepatoprotective effect on the liver by reducing inflammation and dysplasia, and, probably, by reducing oxidative stress.

Acknowledgements: Supported by the RFBR (No. 16-04-00356A, 18-04-00417A). 